\title{
Comparison of base-line and chemical-induced transcriptomic responses in HepaRG and RPTEC/TERT1 cells using TempO-Seq
}

\author{
Alice Limonciel ${ }^{1,3} \cdot$ Gamze Ates $^{2} \cdot$ Giada Carta $^{1,3} \cdot$ Anja Wilmes $^{1,3} \cdot$ Manfred Watzele $^{4} \cdot$ Peter J. Shepard $^{5}$. \\ Harper C. VanSteenhouse ${ }^{5} \cdot$ Bruce Seligmann $^{5} \cdot$ Joanne M. Yeakley $^{5} \cdot$ Bob van de Water $^{6} \cdot$ Mathieu Vinken $^{2}$. \\ Paul Jennings ${ }^{1,3}$
}

Received: 18 August 2017 / Accepted: 4 July 2018 / Published online: 14 July 2018

(c) The Author(s) 2018

\begin{abstract}
The utilisation of genome-wide transcriptomics has played a pivotal role in advancing the field of toxicology, allowing the mapping of transcriptional signatures to chemical exposures. These activities have uncovered several transcriptionally regulated pathways that can be utilised for assessing the perturbation impact of a chemical and also the identification of toxic mode of action. However, current transcriptomic platforms are not very amenable to high-throughput workflows due to, high cost, complexities in sample preparation and relatively complex bioinformatic analysis. Thus, transcriptomic investigations are usually limited in dose and time dimensions and are, therefore, not optimal for implementation in risk assessment workflows. In this study, we investigated a new cost-effective, transcriptomic assay, TempO-Seq, which alleviates the aforementioned limitations. This technique was evaluated in a 6-compound screen, utilising differentiated kidney (RPTEC/TERT1) and liver (HepaRG) cells and compared to non-transcriptomic label-free sensitive endpoints of chemical-induced disturbances, namely phase contrast morphology, xCELLigence and glycolysis. Non-proliferating cell monolayers were exposed to six sub-lethal concentrations of each compound for $24 \mathrm{~h}$. The results show that utilising a 2839 gene panel, it is possible to discriminate basal tissue-specific signatures, generate dose-response relationships and to discriminate compound-specific and cell typespecific responses. This study also reiterates previous findings that chemical-induced transcriptomic alterations occur prior to cytotoxicity and that transcriptomics provides in depth mechanistic information of the effects of chemicals on cellular transcriptional responses. TempO-Seq is a robust transcriptomic platform that is well suited for in vitro toxicity experiments.
\end{abstract}

Keywords HepaRG $\cdot$ RPTEC/TERT1 $\cdot$ TempO-Seq $\cdot$ Stress responses $\cdot$ Dedifferentiation

Alice Limonciel and Gamze Ates are equally contributing first authorship. Paul Jennings and Mathieu Vinken are joint corresponding authors.

Electronic supplementary material The online version of this article (https://doi.org/10.1007/s00204-018-2256-2) contains supplementary material, which is available to authorized users.

Mathieu Vinken

mvinken@vub.ac.be

$\triangle$ Paul Jennings

p.jennings@vu.nl

1 Division of Molecular and Computational Toxicology, Amsterdam Institute for Molecules, Medicines and Systems, Vrije Universiteit Amsterdam, De Boelelaan 1108, 1081 HZ Amsterdam, The Netherlands

2 Department of In Vitro Toxicology and Dermato-Cosmetology, Vrije Universiteit Brussel, Laarbeeklaan 103, 1090 Brussels, Belgium

\section{Introduction}

A distinct advantage of in vitro techniques over whole animal models is their applicability to mechanistic investigations and the ability to use cells of human origin.

3 Division of Physiology, Department of Physiology and Medical Physics, Medical University of Innsbruck, 6020 Innsbruck, Austria

4 Roche Diagnostics GmbH, Nonnenwald 2, 82377 Penzberg, Germany

5 BioSpyder Technologies, Inc., 5922 Farnsworth Ct Ste 102, Carlsbad, CA 92008, USA

6 Division of Toxicology, Leiden Academic Center for Drug Research, Leiden University, PO Box 9503, 2300 RA Leiden, The Netherlands 
Mechanistic-based assays are rapidly becoming the cornerstone of contemporary toxicological investigations, driven by advances in genetic analysis and associated omic methodologies. Transcriptomics has been a key tool in allowing a better understanding of how the cellular program is altered in response to stress situations (Jennings et al. 2013). However, whole genome arrays are still too expensive to be considered for routine use. Additionally, RNA sample preparation and post-analysis is cumbersome when sample numbers are large. Thus, multiple compound concentrations and/or temporal effects are seldom studied in transcriptomic investigations and toxicogenomic data is often limited in dimension (Wilmes et al. 2011, 2013). Where complete dose responses were conducted, for example, by Waldmann et al., the chemical-induced alterations in transcriptomic expression were shown to manifest at concentrations well below alterations in cell viability (Waldmann et al. 2014). Thus, if transcriptomic assays become cheaper and easier to perform, it is likely to become a dominant methodology for hazard and risk assessment, due to the wealth of mechanistic information it provides.

In the present study we investigated the utility of a new, cost-effective technique templated oligo assay with sequencing readout (TempO-Seq) that solves both the cost and throughput issues (Grimm et al. 2016; Yeakley et al. 2017). TempO-Seq is an NGS library preparation method that is based on ligation of detector oligos that are hybridized directly to RNA targets, with the subsequent addition of sample barcodes and sequencing adapters by PCR amplification. In addition, there is no RNA extraction step required. The detector oligos are chimeric and contain a sequence specific to the targeted RNA as well as a sequence in common among the detector oligos for universal primers. TempOSeq thus allows cost-effective, simultaneous quantification of specific mRNA targets without the need to isolate RNA. In this particular study, we utilised a probe set identifying 2839 genes which is a combination of a previously selected gene panel (Mav et al. 2018) supplemented with knowledgebased cellular stress response-related genes identified by the experiences of the academic groups in this publication. The entire gene set utilised is provided in Table $\mathrm{S} 1$. The Mav et al. gene-set was developed by the U.S. Tox 21 Federal collaboration program and represents a data-driven strategy of sentinel genes (selected for biological diversity, maximal information content, and widespread pathway coverage), which was also augmented using a knowledge-driven selection of additional genes (Mav et al. 2018).

Two of the major target organs for systemic toxicology are the liver and kidney. While renal and hepatic in vitro systems are often run within the same project umbrella, they are not usually challenged with the same compounds at the same concentrations. This makes it difficult to compare data and draw conclusions pertaining to tissue-specificity of responses and tissue-specific biomarkers. Here we utilised a human renal and human hepatic cell line (RPTEC/ TERT1 and HepaRG, respectively). RPTEC/TERT1 and HepaRG are considered the most differentiated and stable cell lines currently available for their respective tissues and thus the most applicable for in vitro toxicological investigations (Guillouzo et al. 2007; Doktorova et al. 2013; Aschauer et al. 2013, 2015). Both cell types were used for drug exposures under serum-free conditions and under differentiated non-proliferating monolayer conditions. The cells were exposed to the same six compounds at the same concentrations, measuring the same endpoints (impedance, glycolysis, morphology, and targeted TempO-Seq transcriptomics). The six structurally unrelated compounds selected were ochratoxin A (OTA), potassium bromate $\left(\mathrm{KBrO}_{3}\right)$, cyclosporine A (CsA), acetaminophen (APAP), isoniazid (ISZ) and sodium valproate (VALP). While some of these may be considered preferentially nephrotoxic or hepatotoxic, in reality, many adversely affect both organs and in addition pharmacokinetic properties are likely to play a major role in target organ specificity in vivo.

The present study demonstrates the usefulness of the TempO-Seq methodology, identifies differences in RPTEC/ TERT1 and HepaRG expressed transcriptomes, differences in toxicological responses and identifies chemical- and tissue-specificity of certain gene and pathway responses.

\section{Materials and methods}

\section{Routine cell culture and differentiation}

The hepatic cell line, HepaRG (Guillouzo et al. 2007), was obtained from BioPredic International and the renal proximal tubule cell line, RPTEC/TERT1 (Wieser et al. 2008), from Evercyte GmbH. HepaRG cells were routinely cultured in William's E medium (Gibco 12551032) supplemented with $2 \mathrm{mM}$ Glutamax, $5 \mu \mathrm{g} / \mathrm{ml}$ insulin, $50 \mu \mathrm{M}$ hydrocortisone 21 -hemisuccinate, $100 \mathrm{U} / \mathrm{ml}$ penicillin, $100 \mu \mathrm{g} / \mathrm{ml}$ streptomycin and 9\% Foetal Calf Serum (FCS) (all components were from BioPredic International). RPTEC/TERT1 cells were routinely cultured, differentiated and exposed to chemicals in a 1:1 mix of DMEM (Gibco 11966-025) and Ham's F12 (Gibco 21765-029) (containing a final concentration of $5 \mathrm{mM}$ glucose) and supplemented with $2 \mathrm{mM}$ Glutamax, $10 \mathrm{ng} / \mathrm{ml}$ epidermal growth factor, $36 \mathrm{ng} / \mathrm{ml}$ hydrocortisone, $5 \mu \mathrm{g} / \mathrm{ml}$ insulin, $5 \mu \mathrm{g} / \mathrm{ml}$ transferrin, $5 \mathrm{ng} / \mathrm{ml}$ selenium, $100 \mathrm{U} / \mathrm{ml}$ penicillin and $100 \mu \mathrm{g} / \mathrm{ml}$ streptomycin (Jennings et al. 2009). Cells were cultured in a controlled humidified $37{ }^{\circ} \mathrm{C}, 5 \% \mathrm{CO}_{2}$ environment. Cells were routinely passaged once a week in trypsin EDTA. HepaRG cells were differentiated according to Biopredic's 6 day protocol (Biopredic International 2017). Briefly, cells were seeded on 
Table 1 Concentrations of chemicals used with vehicle and ordering information

\begin{tabular}{|c|c|c|c|c|c|c|}
\hline \multirow[b]{2}{*}{ Name } & \multicolumn{6}{|c|}{ Tested concentrations in $\mu \mathrm{M}$} \\
\hline & Ochratoxin A & Potassium Bromate & Cyclosporine A & $\mathrm{Na}$ valproate & Acetaminophen & Isoniazid \\
\hline Short name & OTA & $\mathrm{KBrO}_{3}$ & CsA & VALP & APAP & ISZ \\
\hline \multicolumn{7}{|l|}{ Dilution } \\
\hline 0 & 0 & 0 & 0 & 0 & 0 & 0 \\
\hline 1 & 0.001 & 80 & 0.05 & 8 & 8 & 8 \\
\hline 2 & 0.01 & 400 & 1 & 40 & 40 & 40 \\
\hline 3 & 0.13 & 800 & 5 & 200 & 200 & 200 \\
\hline 4 & 1 & 2000 & 10 & 1000 & 1000 & 1000 \\
\hline 5 & 10 & 4000 & 15 & 5000 & 5000 & 10,000 \\
\hline Pre-stock & $2.48 \mathrm{mM}$ & - & $15 \mathrm{mM}$ & - & - & - \\
\hline Vehicle & RPTEC/TERT1 Medium & RPTEC/TERT1 Medium & DMSO & HepaRG medium & HepaRG medium & HepaRG medium \\
\hline Source & Sigma & Sigma & Calbiochem & Sigma & Sigma & Sigma \\
\hline Cat No & O1877 & P7332 & 239835 & P4543 & A7302 & $\mathrm{I} 3377$ \\
\hline
\end{tabular}

$0-5$ are the dilutions, all values are expressed in $\mu \mathrm{M}$ unless otherwise stated

$10 \mu \mathrm{g} / \mathrm{cm}^{2}$ collagen I (Biopredic) coated 12 well or 96 well plates in base William's E medium with GlutaMAX and ADD670 additives (Biopredic). Medium was changed after $24 \mathrm{~h}$ to maintenance/metabolism medium (base medium with ADD620 additives) and renewed after $48 \mathrm{~h}$. Three days later the medium was changed to Induction medium (base medium with ADD650 serum-free additives). RPTEC/ TERT1 cells were differentiated by allowing them to reach confluence and remain in a confluent state for at least 7 days before treatment as previously described (Aschauer et al. 2013).

\section{Chemical exposures}

From the past experiences of the two experienced testing laboratories (i.e., Medical University of Innsbruck, MUI, and the Vrije Universiteit Brussel, VUB), a panel of six unrelated compounds and associated concentrations were selected (Table 1). Differentiated cell monolayers were washed and treated with the compounds in serum-free medium for $24 \mathrm{~h}$. All compounds, except for CsA, were water soluble. Stock solutions of CsA (15 mM) were made in $100 \%$ DMSO, aliquoted and frozen. All final concentrations for CsA had a $0.1 \%$ DMSO content and $0.1 \%$ DMSO was used as a vehicle control. OTA was made up to $2.48 \mathrm{mM}$ in supplemented DMEM/Ham's F12 aliquoted and frozen. All other stocks were generated in either HepaRG complete medium or RPTEC/TERT1 complete medium and used freshly (Table 1).

\section{xCELLigence assay}

xCELLigence experiments were conducted at the MUI laboratory. Cells were seeded in the proprietary E-Plates containing the impedance gold electrodes in $60 \mu \mathrm{l}$ medium and differentiated. Impedance was measured intermittently using the RTCA unit in a cell culture incubator. At time of exposure impedance was measured every $5 \mathrm{~min}$ for the 24-h duration. Cell index (CI) was normalised to the impedance before measurement per well.

\section{Temp0-Seq assay}

Three separate batches of HepaRG and RPTEC/TERT1 were cultured in separate medium stocks and seeded and differentiated in 12-well plates. The HepaRG and RPTEC/ TERT1 experiments were conducted at the VUB and the MUI, respectively. The three biological replicates of differentiated cells were exposed to chemicals, medium controls and $0.1 \%$ DMSO controls for $24 \mathrm{~h}$ in serum-free medium. Morphology was documented by phase contrast microscopy (Fig. 2). Wells were washed in $1 \mathrm{ml}$ PBS (DPBS, Gibco, 14190-094) and lysed in $750 \mu$ of $1 \times$ BioSpyder lysis buffer. Lysates were frozen at $-80{ }^{\circ} \mathrm{C}$ and shipped to BioSpyder technologies on dry ice where the TempO-Seq assay was conducted.

Cellular extracts were harvested for TempO-Seq analysis to quantify the 3050 probe set representing 2839 genes (Table S1). A pair of detector oligos were annealed to adjacent $25 \mathrm{nt}$ sequences in the target RNA, after which excess oligos were digested with a nuclease, and remaining oligos were ligated (Yeakley et al. 2017). This process occurred as a homogenous assay through progressive dilution to ensure enzyme compatibility and was, therefore, free of 
the need for bead-based clean-up or poly (A) + selection. Furthermore, the assay did not require RNA purification or cDNA synthesis. Once ligated, the detector oligos acted as templates in a PCR-based amplification to add sequencing adapters for an Illumina instrument and sample barcodes. Since all the detector oligos share the same pair of primer landing sites, the assay could be multiplexed with respect to the RNA targets, but still single plex with respect to the primers. The PCR primers both include a 9-mer index sequence, different for the primers used to amplify each sample, that resulted in dual index sample barcodes, allowing pooling post-PCR of up to 384 samples in 1 library. The number of samples per library depends on the sequencing depth achievable for a given instrument and the read depth desired. For a detector pool of $\sim 3 \mathrm{~K}$ targets, between $0.5 \mathrm{M}$ and $3 \mathrm{M}$ reads per sample should give correlations of $\mathrm{R}^{2}=\sim 0.97$ for technical replicates of $100 \mathrm{ng}$ purified total RNA. Each sample fastq file was aligned against the TempO-Seq transcriptome using the Bowtie aligner ( $\mathrm{Li}$ and Durbin 2009). The output of this analysis generated a table of counts per gene per sample.

\section{Lactate assay}

Supernatant samples from the same TempO-Seq run were centrifuged at $150 \mathrm{~g}$ for $5 \mathrm{~min}$ to remove free floating cells. The supernatant was frozen at $-20{ }^{\circ} \mathrm{C}$ until assay. Samples from the VUB lab were transferred to the MUI lab for assay. In a 96 well microtitre plate $10 \mu \mathrm{l}$ supernatant medium was incubated with $90 \mu \mathrm{l}$ lactate reagent buffer ( $86 \mathrm{mM}$ Triethanolamine $\mathrm{HCl}, 8.6 \mathrm{mM}$ EDTA. $\mathrm{Na}_{2}$, $33 \mathrm{mM} \mathrm{MgCl} 2,326 \mu \mathrm{M} \mathrm{N}$-methylphenazonium methyl sulphate (PMS), $790 \mu \mathrm{M}$ p-iodonitrotetrazolium violet (INT), $3.37 \mathrm{mM} \beta$-NAD, 7\% ethanol, 0.4\% Triton-X-100, $4 \mathrm{U} / \mathrm{ml}$ Lactate Dehydrogenase) for approximately $7 \mathrm{~min}$ at room temperature, as previously described (Limonciel et al. 2011). Optical density was measured in a Tecan Infinite M200 at $490 \mathrm{~nm}$. An 8-point calibration curve starting with $25 \mathrm{mM}$ lactate was used to determine actual concentrations. To avoid confusion we wish to clarify that this assay measures the metabolite lactate, not the activity of the lactate dehydrogenase enzyme, which is often used as a cell viability (plasma membrane integrity) assay.

\section{Data analysis and visualisation}

TempO-Seq sample outliers were assessed for the two cell types independently and identified using both the Tukey's and Grubb's outlier tests. No outliers were uncovered in this sample set. Normalization and differential expression were performed using the DESeq2 package (Love et al. 2014). To perform differential expression analysis, each treatment condition was paired with the appropriate control and the counts for each sample were then normalized using the DESeq2 estimateSizeFactors function. Differential expression of each treatment relative to its respective control was measured using the Wald test. Probes with Benjamin Hochberg adjusted $p$ values $\leq 0.05$ were considered significantly differentially expressed.

Heat maps were generated using the conditional formatting function of Microsoft Excel (version 1803). Genes were assigned to ATF4, Nrf2 or p53 pathways using information from several published sources (Riley et al. 2008; Limonciel et al. 2015). For a complete list of pathway allocations and sources see Table S3. Toxicological Prioritization Index (ToxPi) visualisations were generated using the National Center for Computational Toxicology, U.S. EPA's ToxPi software version 2.0 (Reif et al. 2013; Marvel et al. 2018). The linear algorithm was used for all Pis, with equal weightings. The complete input file for the ToxPi software is given in Table S5.

\section{Statistical analysis}

For concentration dependent analysis a one-way ANOVA with Dunnett's post-test vs control (lactate and individual genes) was conducted. For time and concentration analysis a two-way ANOVA was used with a Sidak's post-test (xCELLigence). Both were generated using GraphPad Prism version 6 . See legends for specific details.

\section{Results}

The control samples, consisting of three medium controls and three $0.1 \%$ DMSO treated controls per cell line, were used to compare the baseline gene expression of differentiated HepaRG and RPTEC/TERT1 cells (Fig. 1 and Table S2). While RPTEC/TERT1 and HepaRG exhibited a similar expression of the majority of genes (Fig. 1a inset, Table S2A), 519 genes had significantly different basal expression levels in the two cell lines (Fig. 1 main, Fig. $1 \mathrm{~b}$ and Table S2B). HepaRG exhibited significantly higher expression of genes encoding plasma proteins, including albumin (ALB), haptoglobin (HP), transthyretin (TTR), alpha fetoprotein (AFP), alpha-2-macroglobulin (A2M), apolipoproteins (APOA1, APOC1, APOE), fibrinogen (FGG, FGB) and complement proteins (C3, C1R and $\mathrm{CFH}$ ), genes involved in xenobiotic metabolism, including cytochrome P450s (CYP3A4, CYP3A5, CYP2E1), $\mathrm{N}$-acetyltransferase 2 (NAT2), carboxylesterase 1 (CES1), and other known liver-specific genes, including fatty acid binding protein 1 (FABP1), orosomucoid 1 and 2 (ORM1, ORM2), ABCC2, alcohol dehydrogenase (ADH1A and 1B), SLCO1B1, SLCO2B1, bile acid-CoA:amino acid 
Fig. 1 Comparison of HepaRG and RPTEC/TERT1 transcriptome. From a total of 3050 probes (inset a), probes were filtered for significant difference between the in vitro models $(p$ value $<0.001)$ and with a coefficient of variance greater than 0.6. a Plot of HepaRG mRNA values against the corresponding RPTEC/TERT1 values. b Most abundant 15 genes from the 105 genes which are exclusively expressed in 1 model. All values are given in Table S2A and S2B
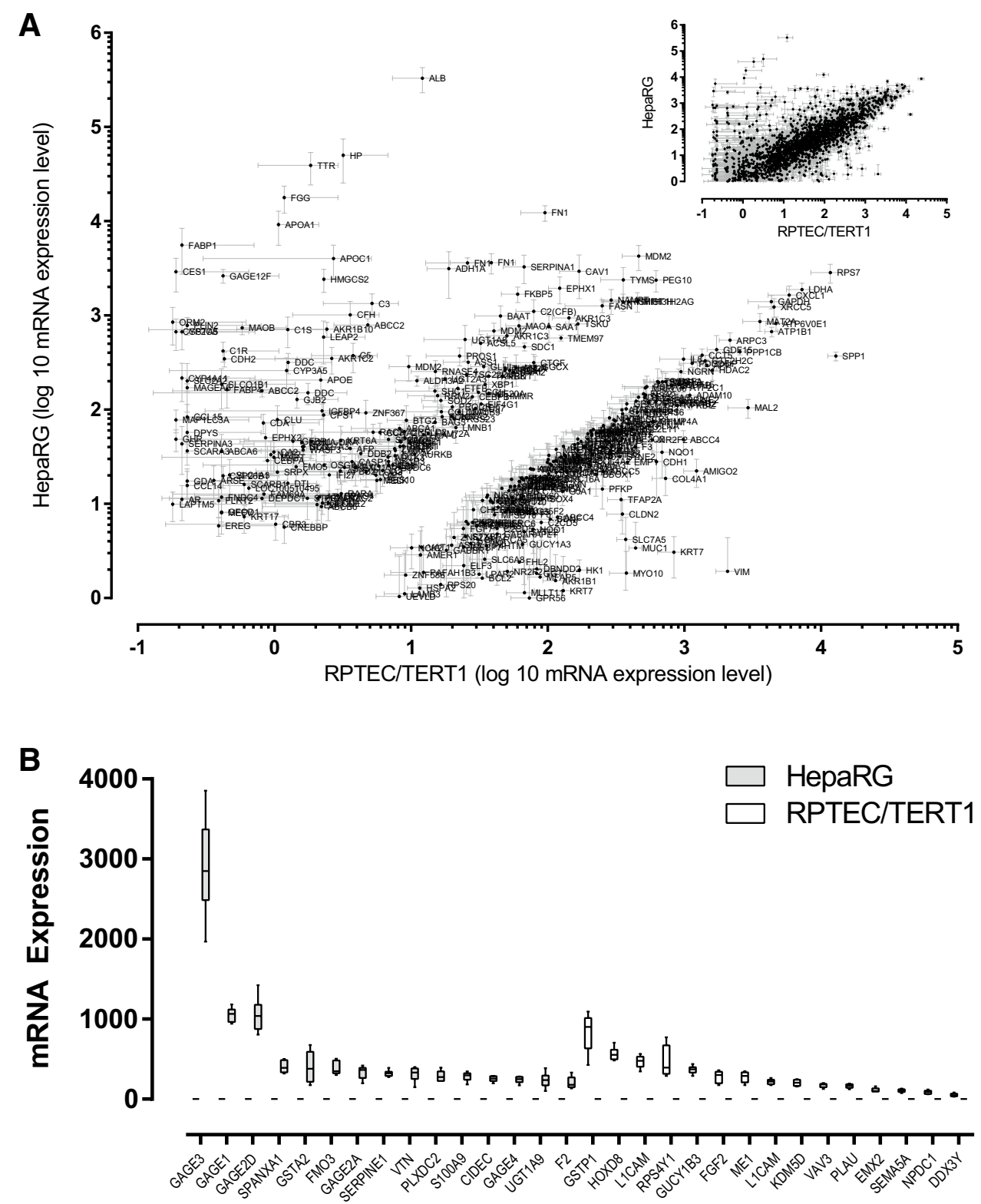

$\mathrm{N}$-acyltransferase (BAAT), genes involved in the urea cycle Arginase 1 (ARG1) and the transcription factor hepatocyte nuclear factor 4 alpha (HNF4A). HepaRG, compared to RPTEC/TERT1 cells also highly express Epiregulin (EREG) and several genes associated with cancer, many belonging to the GAGE family (GAGE $-1,-2,-3,-4$ and -12 ).

RPTEC/TERT1 cells exhibited a higher expression of genes including the nephrogenic transcription factor PAX8, the tight junction protein claudin 2 (CLDN2), SLC7A5 and SLC3A2 (the genes encoding the proteins for the large neutral amino acid transporter LAT1), the brush border enzyme gamma glutamyl transferase (GGT1), collagen 4A (COL4A), methionine adenosyltransferase 2A (MAT2A), secreted phosphoprotein 1 (SPP1), mal, T-cell differentiation protein 2 (MAL2), ATP binding cassette subfamily $\mathrm{C}$ members (ABCC 4 and 5), phosphofructokinase (PFKP), gamma-butyrobetaine hydroxylase 1 (BBOX1), vimentin (VIM), ATPase $\mathrm{Na}+/ \mathrm{K}+$ transporting subunit beta 1 (ATP1B1), the proton pump (ATP6VOE1), glyceraldehyde 3-phosphate dehydrogenase (GAPDH), ribosomal protein S7 (RPS7), amyloid beta precursor protein (APP), myosin $\mathrm{X}$ (MYO10), NAD(P)H quinone dehydrogenase 1 (NQO1), mucin 1 (MUC1), adhesion molecule with Ig like domain 2 (AMIGO2) and plasminogen activator urokinase (PLAU).

Upon exposure to the selected compounds, temporal alterations in impedance utilising the xCELLigence system showed an initial spike after all treatments in both cell lines, presumably due to the manipulation of the cells outside the 
A RPTEC/TERT1: impedance

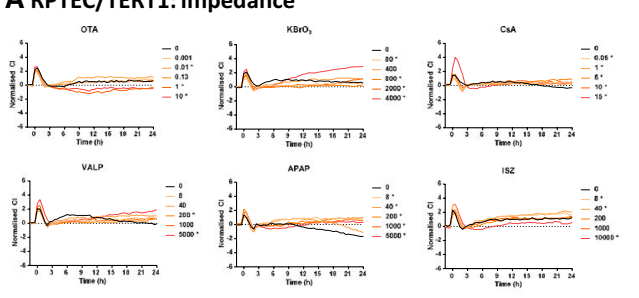

B HepaRG: impedance
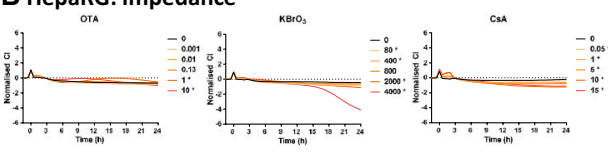

132
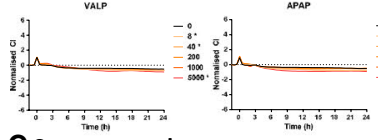

C Supernatant lactate
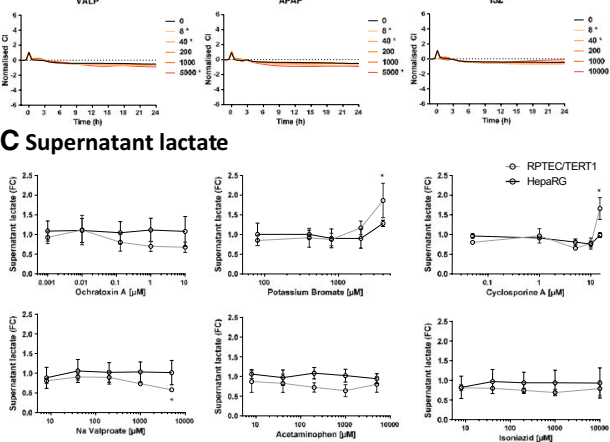

Fig. 2 xCELLigence, lactate production and morphology after $24 \mathrm{~h}$ single application exposures. a, b Impedance measurements utilising the $\mathrm{xCELLigence} \mathrm{system} \mathrm{with} \mathrm{the} \mathrm{same} \mathrm{concentrations} \mathrm{as} \mathrm{utilised}$ for the transcriptomics study. The values are mean from three biological replicates. *Indicates a significant difference using a two-way ANOVA, using a Sidak's post-test with a significant cut off of 0.05 . c

incubator (Fig. 2a, b). The xCELLigence apparatus measures net impedance of each well, reported as Cell Index (CI), with high temporal resolution (Kustermann et al. 2013; Kho et al. 2015). CI is dependent on the amount of cells attached to the well, the forces at which they bind and the net volume of the cells per well and is considered a sensitive labelfree viability assay (Ke et al. 2011). RPTEC/TERT1 cells exhibited an oscillating CI, compared to the smooth CI of HepaRG cells. RPTEC/TERT1 monolayers due to vectorial solute and water transport, form raised areas called, domes (Wieser et al. 2008; Aschauer et al. 2013). Where a dome forms, the cells are no longer in contact with the electrode and thus do not contribute to impedance. Since, domes are dynamic and are formed and collapse overtime, the CI pattern appears ruffled. The strongest compound effect observed in RPTEC/TERT1 was with $4000 \mu \mathrm{M} \mathrm{KBrO}_{3}$. This condition resulted in an increase of $\mathrm{CI}$, beginning at 9-h exposure (Fig. 2a), which is likely due to toxicity induced decrease in dome formation. In HepaRG $4000 \mu \mathrm{M} \mathrm{KBrO}_{3}$ also showed the strongest effect, with a decrease in CI starting at 15 -h exposure (Fig. 2b). Enhanced glycolysis, as measured by supernatant lactate, was observed in RPTEC/TERT1 cells only, and only in two conditions: CsA $15 \mu \mathrm{M}$ and $\mathrm{KBrO}_{3}$
Supernatant lactate from the same cultures utilised in the transcriptomic study. Values are mean fold control $(\mathrm{FC}) \pm$ standard deviation. *Indicates a significant difference using a one-way ANOVA, using a Dunnett's post-test with a significant cut off of 0.05 . d, e Phase contrast morphology of cells with the highest concentration of compounds

$4000 \mu \mathrm{M}$ (Fig. 2c). VALP caused a slight decrease in lactate production at the highest concentration in RPTEC/TERT1 cells (Fig. 2c). Phase contrast morphology shows a typical representation of differentiated RPTEC/TER1 cells and differentiated HepaRG cells; with a complete monolayer, cobble stone morphology and dome formation in the RPTEC/ TERT1 controls (Fig. 2d) and a mixture of compact hepatocyte-like cells and biliary epithelial-like cells for HepaRG cells (Fig. 2e). Morphological aberrations were observed only with $\mathrm{KBrO}_{3}$ at $4000 \mu \mathrm{M}$ in RPTEC/TERT1 cells, with small holes visible in the monolayer (Fig. 2d, e).

Figure 3 shows the read counts, differentially expressed probes (DEP) and a heat map of genes which were differentially expressed compared to controls in at least 2 of the 60 conditions (i.e., cell type, compounds, and concentration). The treatments had no discernible effect on read counts (Fig. 3a). There was, however, a concentration-dependent increase (i.e., an increase in at least two consecutive concentrations) in the number of DEPs for all compounds except ISZ. OTA had the strongest impact on DEPs, followed by $\mathrm{KBrO}_{3}$, CsA, VALP, APAP, and ISZ. (This does not reflect the potency ranking as different concentrations were used.) Based on the first concentration with an increase in DEP, 
Fig. 3 Effect of compounds on read counts (a), differential gene expression per treatment (b) and individual differential gene expression (c). The total read counts $(\times 1000)$ are plotted versus concentration per cell model. $\mathbf{b}$ The number of total differentially expressed genes (with a significance cut off Benjamini Hochberg adjusted $p>0.05$ ), are plotted versus concentration per cell model. The numeric values are given in the tables. c Heat map of $\log 2$ fold control data from 1514 genes that were significantly altered in 2 or more conditions. The heat map is sorted by the sum across all conditions per gene. $\mathrm{R}$ is RPTEC/ TERT1, H is HepaRG. The red colour indicates increased and blue decreased, where white is unchanged. Where the $\log 2$ fold control could not be calculated (i.e., a division by zero error where all values zero in the control) the value is also represented by a white. The values at the bottom of the heat map are the sum of the DEGs over the 5 concentrations per cell line (maximum possible is 7570)

The values behind the heat map with the gene annotations are given in Table $\mathrm{S} 4$
A
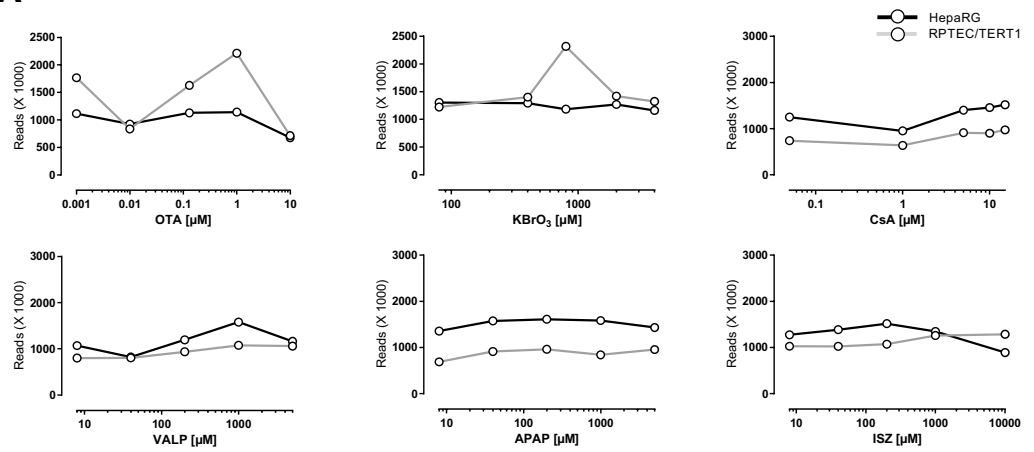

B
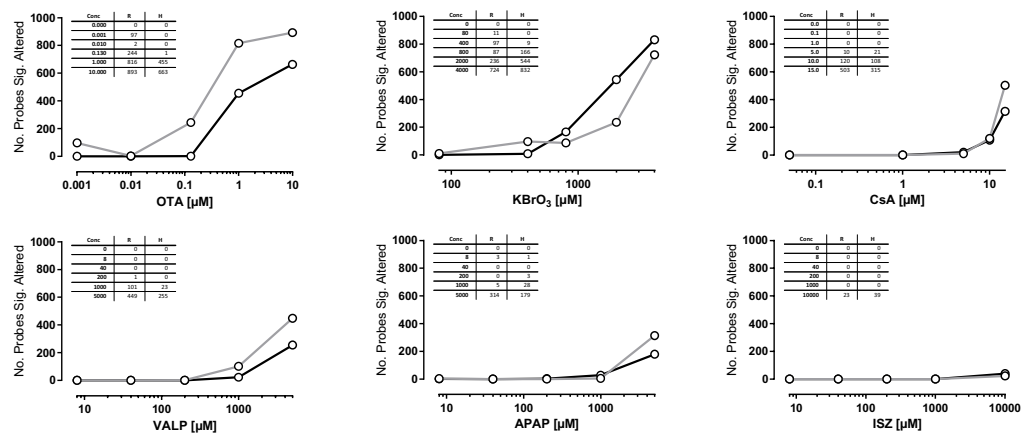

C

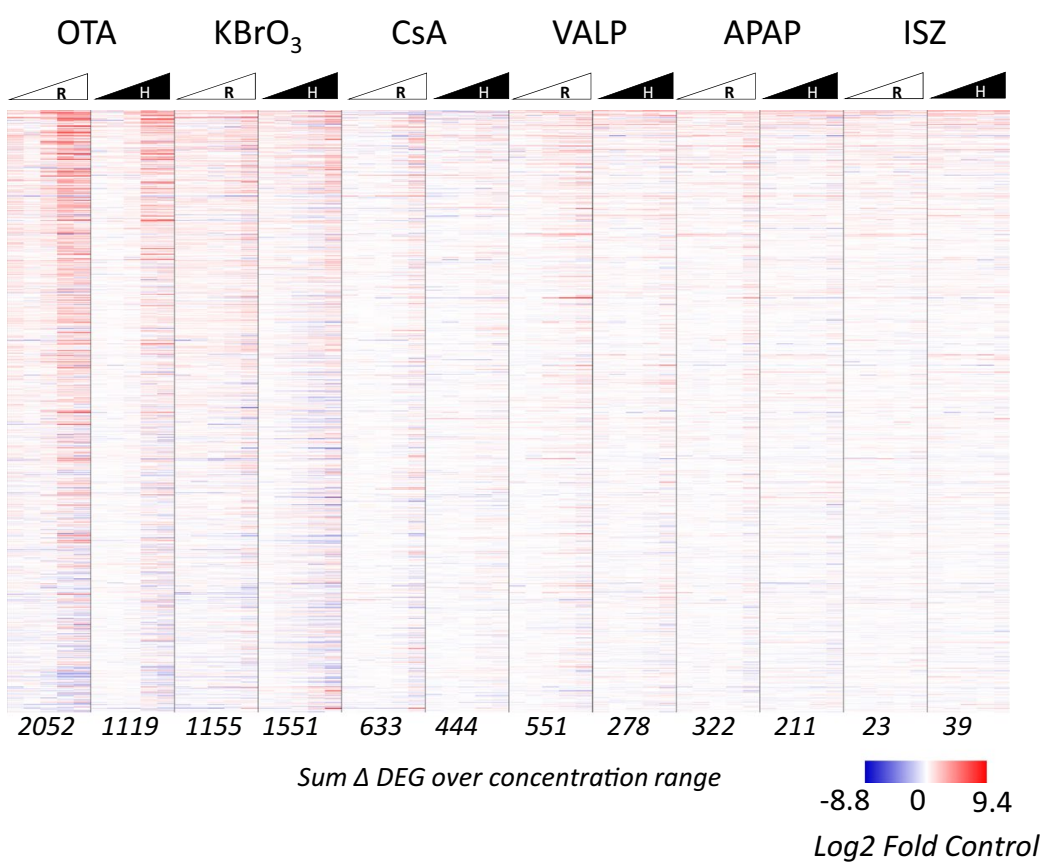

RPTEC/TERT1 were more sensitive to OTA and $\mathrm{KBrO}_{3}$, and sensitivities for the rest of the compounds were similar.

The data are also visualised using ToxPi with the slices representing DEP, genes in the ATF4 (major branch of the unfolded protein response), Nrf2 and p53 pathway (Fig. 4). It should be noted that the ToxPi charts do not show directionality of the genes in the pathway, just overall impact.
Utilising the hierarchical clustering feature, three main clusters were apparent (Fig. 4). The yellow cluster represents the concentrations that had the highest impact on the four parameters and includes the two highest concentrations of OTA and $\mathrm{KBrO}_{3}$ for both cell types (OTA_H4,H5, OTA $\left.\mathrm{R} 4, \mathrm{R} 5, \mathrm{KBrO}_{3} \_\mathrm{H} 4, \mathrm{H} 5, \mathrm{KBrO}_{3} \mathrm{R} 4, \mathrm{R} 5\right)$ and the highest concentration of CsA in RPTEC/TERT1 cells (CsA_R5). The 
Fig. 4 Representation of compound-induced differentially expressed genes using ToxPi visualisations. The ToxPi v 2 software was used to generate the diagrams. The top panel shows a full pie with its colour codes and gene numbers each slice uses the linear model. DEP is differentially expressed probes. The numbers for $\mathrm{p} 53$, Nrf2 and ATF4 represent the no. of genes allocated to those pathways (see Table S3). On the right of the top panel is a Hierarchical Clustering using ward.D2. Labelling of samples is compound code, the dilution (as per Table 1), R for RPTEC/ TERT1 and $\mathrm{H}$ for HepaRG The lower panels show the ToxPis per cell type, compound and concentrations, where numbers represent the $\mu \mathrm{M}$ concentration of the corresponding compound. The ToxPi data are given in Table S5.

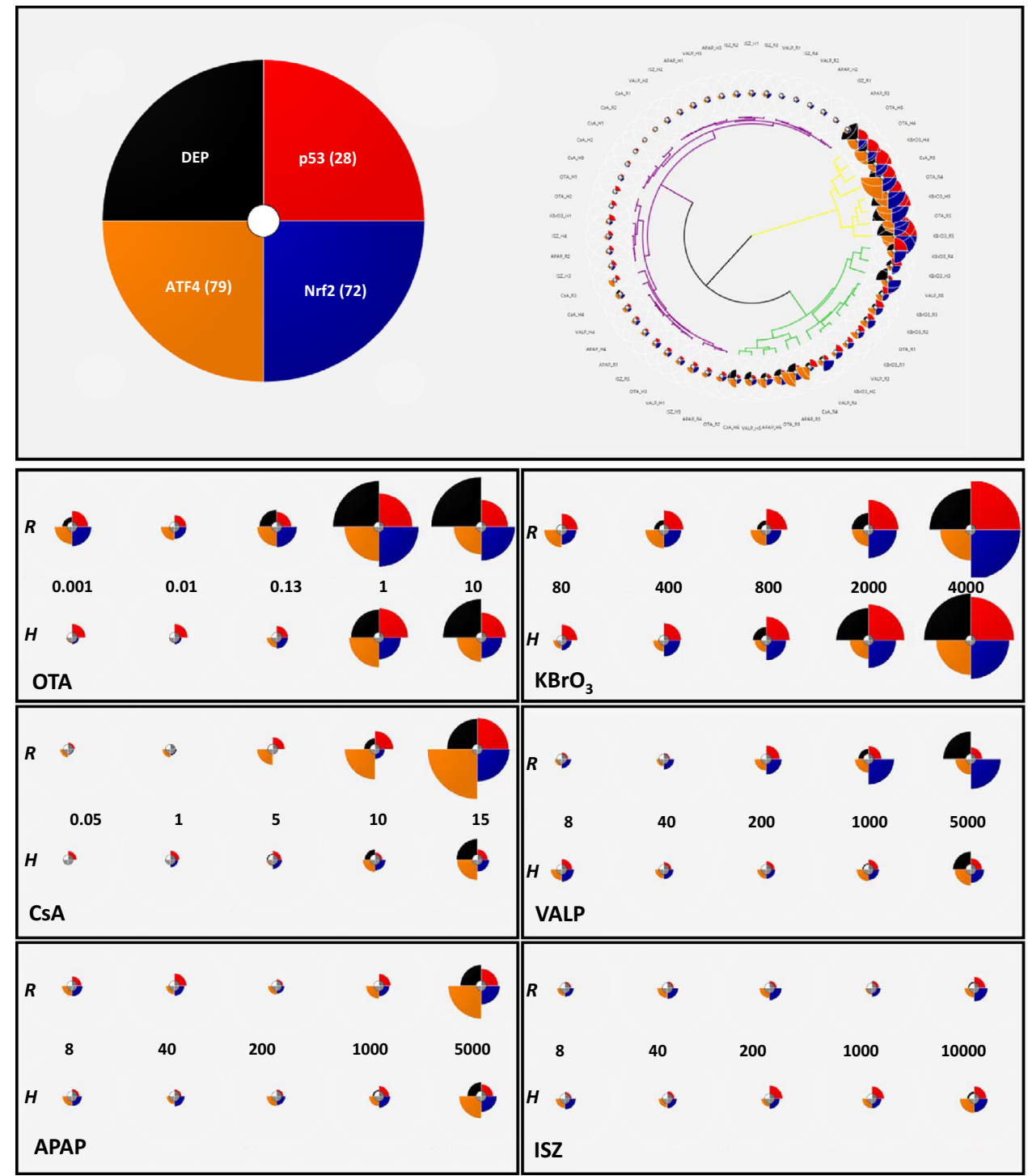

green cluster represents the conditions that had a medium impact and the purple cluster contains mild to zero impact. All of the ISZ compounds were in the green cluster. In the lower panels of Fig. 4 the ToxPis are organised per compound and cell type. OTA had a strong impact on all three pathways, but the impact was larger in RPTEC/TERT1 cells. $\mathrm{KBrO}_{3}$ had a strong impact the Nrf2 and p53 pathways. Both CsA and APAP had a stronger impact on the ATF4 pathway and this was larger in RPTEC/TERT1 cells. In RPTEC/ TERT1 cells VALP had a prominent effect only on the Nrf2 pathway. None of the selected pathways were impacted on by ISZ in either cell type.

Quantitative data for 27 selected representative genes is given graphical form as non-transformed and in heat maps as $\log 2$ fold control transformed data (Fig. 5). The first panel of nine represent genes in the Nrf2 (HMOX1, SRXN1 and GCLM), p53 (GADD45A, CDKN1A, SFN) and ATF4 pathways (ASNS, TRIB3, DDIT3) (Fig. 5a). For the Nrf2 genes, HMOX1 was more responsive in RPTEC/ TERT1 cells, while SRXN1 and GCLM showed similar levels of induction (Fig. 5a). $\mathrm{KBrO}_{3}$ was the strongest inducer of these genes. At the highest concentration of OTA HMOX1 was significantly decreased in RPTEC/ TERT1 and GCLM significantly decreased in both cell types. For the p53 genes, GADD45A, CDKN1A and SFN showed similar levels of induction in both cell types and again $\mathrm{KBrO}_{3}$ was the highest inducer. OTA decreased SFN at the highest 2 concentrations in HepaRG cells. For the ATF4 genes, both TRIB3 and DDIT3 were more responsive in RPTEC/TERT1, whereas ASNS showed a similar level of maximum induction in both cell types. CsA, particularity in RPTEC/TERT1 cells, was the highest inducer of these three genes, but the highest concentration of APAP also resulted in a significant increase in ATF4 genes in both cell types. OTA decreased TRIB3 in both cell types. 
A Stress Response Pathways

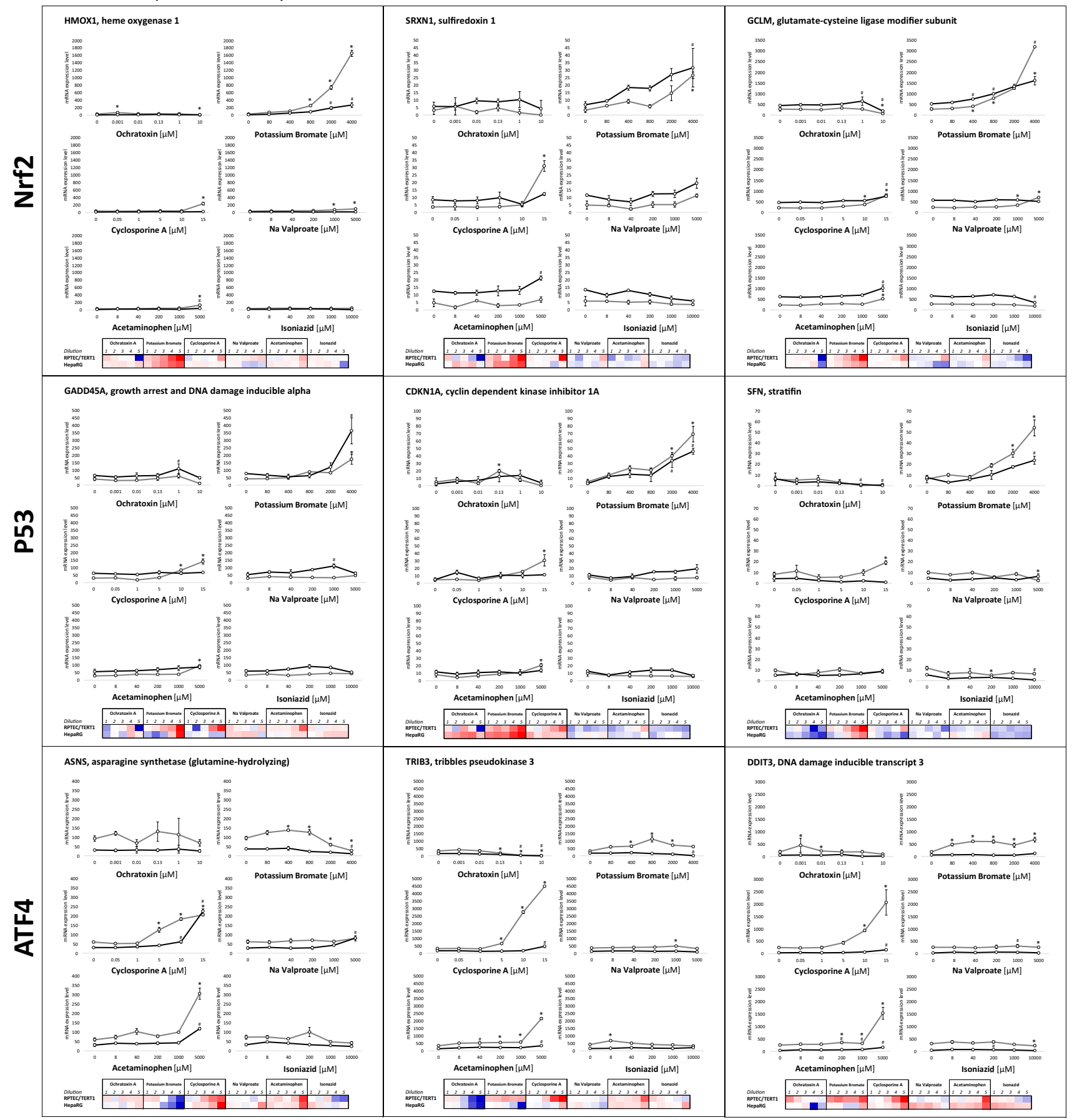

Fig. 5 Concentration response relationships of selected genes, representing a stress response pathways, b HepaRG-specific and $\mathbf{c}$ RPTEC/ TERT1-specific. In the graphs the values are expressed as mean mRNA expression \pm SEM. In the heat map values represent the mean $\log 2$ fold control with red representing the highest value and blue the

Examples of HepaRG responsive genes are given in Fig. 5b. CYP3A4, EREG, A2M, ARG1, HNF4A, ADH1A were unresponsive in RPTEC/TERT1 cells. AFP, BAAT and HPR showed some mild induction in certain exposures in lowest. White represents either 0 or absent. Statistical significance to control $(p<0.05)$ is denoted by * for RPTEC/TERT1 and \# for HepaRG using a one-way ANOVA, with a Dunnett's post-test and significant cut off of 0.05

RPTEC/TERT1. All of these 9 genes were robustly responsive with at least one of the compounds in HepaRG. APAP was a strong inducer of CYP3A4; OTA and $\mathrm{KBrO}_{3}$ were inducers of AFP; while $\mathrm{KBrO}_{3}$ and VALP were inducers of 


\section{B HepaRG specific}

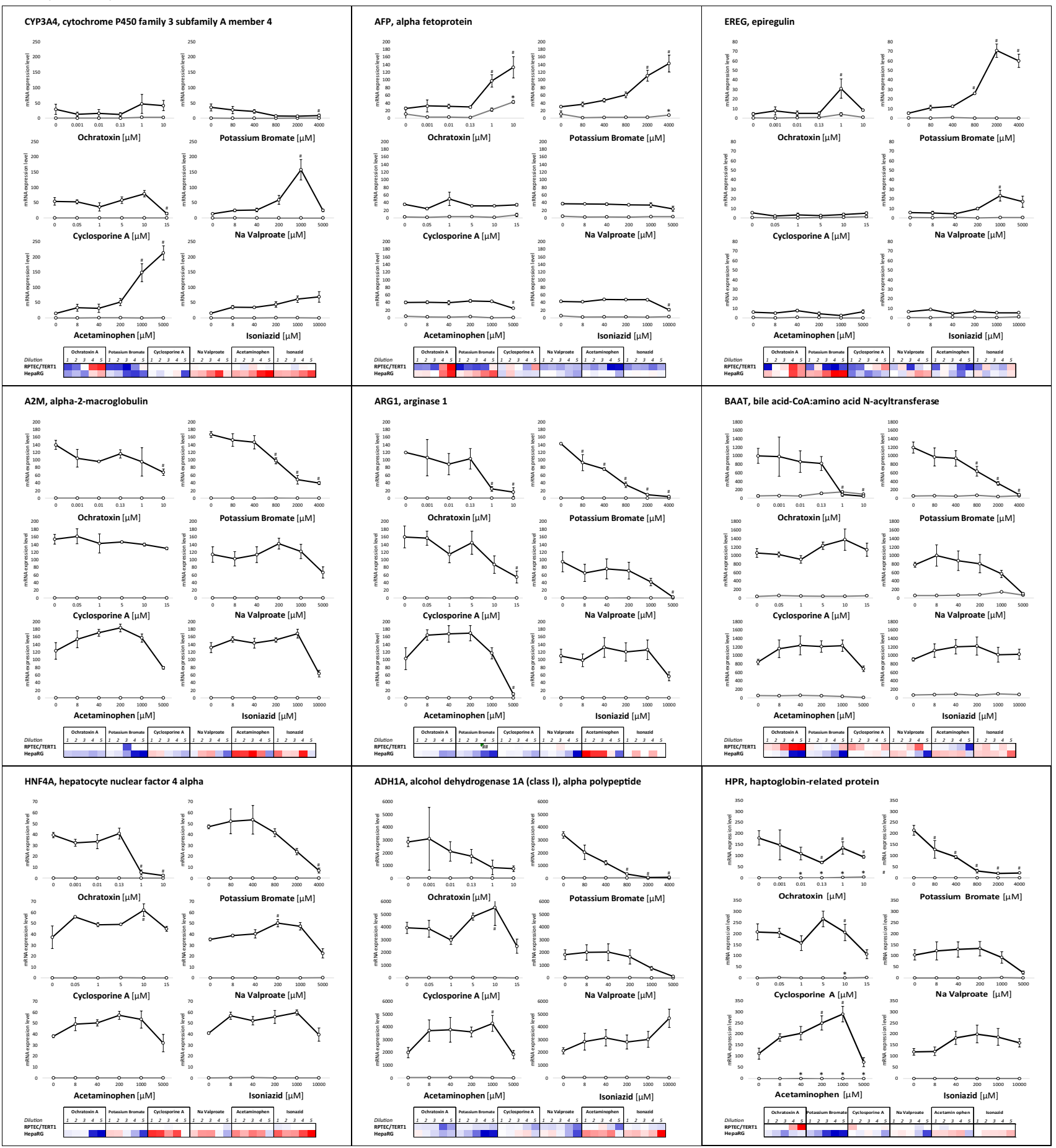

Fig. 5 (continued)

EREG. A2M, ARG1, BAAT, HNF4A, ADH1A and HPR were mostly decreased in the chemical exposures, which was particularly predominant with $\mathrm{KBrO}_{3}$ exposure.

Examples of RPTEC/TERT1 responsive genes are given in Fig. 5c. SPP1, SLC7A5, ME1, BBOX1, and MUC1 were unresponsive in HepaRG cells. APP, CLDN2, ATP1B1 and AMIGO2 showed some mild responses in HepaRG. All of these 9 genes were robustly responsive with at least three of the compounds in RPTEC/TERT1. SPP1 was induced by OTA, $\mathrm{KBrO}_{3}$ and CsA. APP was induced by all compounds 


\section{RPTEC/TERT1 specific}

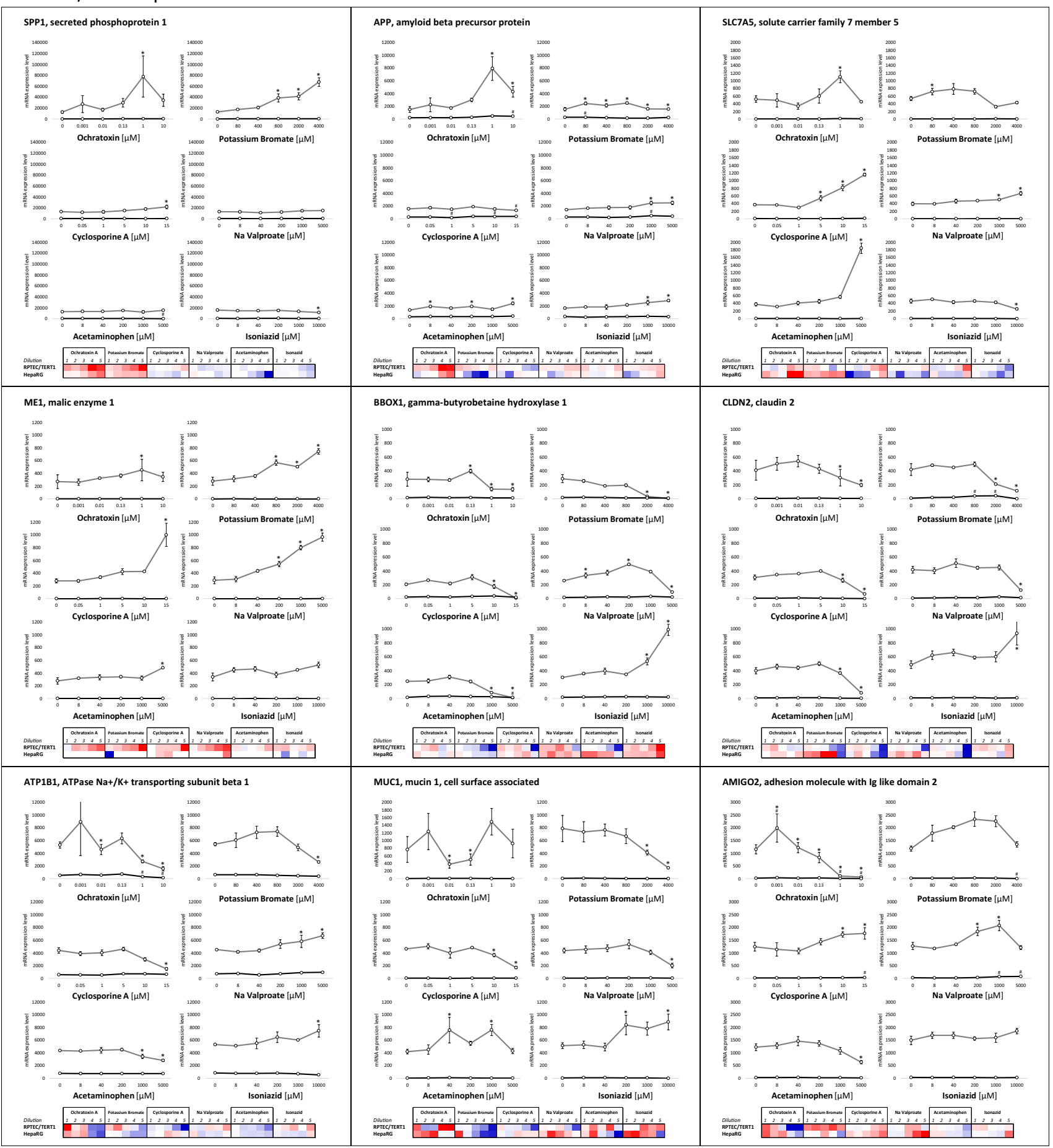

Fig. 5 (continued)

except CsA. SLC7A5, which is also an ATF4 responsive gene, was induced by CsA, APAP and VALP. ME1, which is an Nrf2 responsive gene was induced by $\mathrm{KBrO}_{3}$, CsA and VALP. BBOX1, CLDN2, ATP1B1 and MUC1 were frequently decreased in chemical exposures, however, all were induced by ISZ. AMIGO2 was induced by CsA and VALP (VALP was biphasic), whereas OTA and APAP attenuated AMIGO2. 


\section{Discussion}

The main aim of this study was to investigate the use of the TempO-Seq platform for application to in vitro toxicology. Comparison of the base-line expression of the 2 cell types uncovered a clear separation of 519 genes (Fig. 1 and Table S2B). HepaRG cells differentially expressed genes related to their liver origin including; plasma proteins, metabolising enzymes, and mediators of fatty acid metabolism. There was also high expression of GAGE isoforms and EREG, which likely relates to their cancer origin as compared to the non-cancerous RPTEC/TERT1 cells (Wieser et al. 2008). The RPTEC/TERT1 cell line, differentially expressed genes involved in renal phenotype including; transport, tight junction formation, and energy metabolism. Thus, the targeted gene panel, and the cell types utilised, captured renal and hepatic phenotypes. Challenging the cells with six compounds at six concentrations revealed a clear concentration-response relationship in the total number of differentially expressed probes for all compounds except ISZ. (Here concentration-response is defined as an increase in at least two consecutive concentrations and ISZ had an effect only at the highest concentration). Taking into consideration the concentrations applied, the potency ranking was $\mathrm{OTA}>\mathrm{CsA}>\mathrm{KBrO}_{3}>\mathrm{VALP}>\mathrm{APAP}>\mathrm{ISZ}$. RPTEC/ TERT1 cells were more sensitive to OTA and $\mathrm{KBrO}_{3}$, while both cell lines were similar sensitive to the other compounds. In the cellular assays and from phase contrast morphology only $\mathrm{KBrO}_{3}$ and/or CsA showed a strong effect and only at the highest concentration applied, thus transcriptomic signatures were far more sensitive than these assays.

An advantage of TempO-Seq (and other sequencingbased techniques) is the possibility to use linear mRNA counts. While $\log 2$ control readouts are useful, especially for visualisation, it can also reduce data to sometimes biological meaningless information, especially where untreated values are close to zero. This phenomenon is well represented here in the comparison of the non-transformed and the $\log 2$ transformed data of the HepaRG-specific and RPTEC/TERT1specific compound responses (Fig. 5b and c, graphs vs heat maps).

Several mechanistic aspects of compound-induced effects could be garnered from the TempO-Seq analysis. OTA, a nephrotoxic carcinogenic mycotoxin in rodents (IARC 1993; Zhang et al. 2016), was the most potent compound used and exhibited a high impact on the selected pathways, i.e., ATF4, Nrf2 and p53 (Figs. 3, 4). However, this impact was uniquely an OTA-induced pathway suppression as none of the nine panel pathway genes were increased with increasing OTA exposures, in fact the majority were decreased by OTA (Fig. 5a). Similar observations have been reported with OTA previously in the context of Nrf2 suppression (Cavin et al.
2006; Boesch-Saadatmandi et al. 2008; Limonciel and Jennings 2013). OTA-induced suppression of stress responses may contribute, or even underlie, its toxicity and carcinogenicity. $\mathrm{KBrO}_{3}$ a group 2B human genotoxic carcinogen, mediated by oxidant activity (DeAngelo et al. 1998; Limonciel et al. 2012), resulted in a strong concentration-dependent activation of the Nrf2 and p53 pathways in both cell types, while ATF4 was to a lesser extent activated. ASNS expression was even attenuated by $\mathrm{KBrO}_{3}$. Cyclosporine A (CsA) is a cyclophilin binding calcineurin inhibitor and potent immunosuppressant, but is also a competitive inhibitor of the bile salt export pump (ABCB11) (Starokozhko et al. 2017). CsA at supra-therapeutic concentrations $(15 \mu \mathrm{M})$ has been shown to injure mitochondria and cause endoplasmic reticulum (ER) disturbances (Wilmes et al. 2013). Here, CsA showed predominately a strong activation of the ATF4 genes, which is in keeping with previous findings (Wilmes et al. 2013). The anti-epileptic compound sodium valproate (VALP) has been linked with liver steatosis and renal proximal tubular dysfunction, potentially via $\beta$-oxidation inhibition and mitochondrial dysfunction (Chang and Abbott 2006; Komulainen et al. 2015; Willebrords et al. 2015; Heidari et al. 2017). Here, VALP resulted in an activation of the Nrf2 pathway in both cell types and induced the ATF4 regulated gene ASNS in HepaRG cells. Acetaminophen (APAP), the widely used analgesic is well documented to cause liver injury in high doses due to formation of the metabolite N-acetyl-p-benzoquinoneimine (NAPQI) and subsequent NAPQI covalent thiol reactivity, glutathione depletion, resulting in proteotoxicity and ER stress (Bessems and Vermeulen 2001). Here, APAP had a prominent effect on ATF4 genes in both cell types. The major target organ of INZ is considered to be the liver, where INZ metabolites are thought to bind covalently to proteins (Hassan et al. 2015; Metushi et al. 2016; Iorga et al. 2017). However, ISZ had the lowest impact here, and we did not observe an impact on any of the pathways investigated.

Many of the cell type-specific genes were also altered in response to compound exposures; 18 of these were selected for deeper analysis, 9 for HepaRG and 9 for RPTEC/TERT1. The hepatic phase I metabolising gene, CYP3A4 was significantly induced in HepaRG by APAP and VALP, the latter having a biphasic response. Both compounds have been previously shown to be CYP3A4 inducers (Feierman et al. 2002; Cerveny et al. 2007). CsA and $\mathrm{KBrO}_{3}$ decreased CYP3A4 expression. CsA suppression of CYP3A4 has also been previously reported (Amundsen et al. 2012). AFP was strongly induced by the carcinogens OTA and $\mathrm{KBrO}_{3}$ in HepaRG cells and to a lesser extent in RPTEC/TERT1 cells. AFP is a well-recognised marker of a hepatic foetal phenotype and is also discussed as a marker of hepatocellular carcinoma (Terentiev and Moldogazieva 2013). EREG, also a cancer associated protein (Riese and Cullum 2014), 
was induced by OTA, $\mathrm{KBrO}_{3}$ and VALP only in HepaRG cells. A2M, ARG1, BAAT, HNFA4, ADH1A and HPR were also affected by compound exposure and were for the most part down regulated. These genes are predominately liver expressed and HNF4A, for example, has been shown to be important in the maintenance of hepatic differentiation, where its overexpression can rescue dedifferentiation processes (Späth and Weiss 1997). Globally the described alterations of these nine genes could be interpreted as compound-induced dedifferentiation of the hepatic phenotype.

SPP1 was induced by OTA, $\mathrm{KBrO}_{3}$ and CsA in RPTEC/ TERT1 cells. The induction of SPP1, which is more predominately expressed in native kidney tissue, has been previously associated with renal and not hepatic injury in rats in vivo (Dadarkar et al. 2010). APP, which has role in neural growth and repair (Dawkins and Small 2014) was induced by all compounds, with the exception of CsA. SLC7A5, is involved in large neutral amino acid reabsorption, is strongly expressed in renal tubules and is also inducible by ATF4 (Han et al. 2013). SLC7A5 was induced by APAP, CsA and VALP. ME1, which is mildly expressed in the kidney in vivo under basal conditions, but is also under the transcriptional regulation of $\mathrm{Nrf2}$ (Wu et al. 2011) was heavily increased by $\mathrm{KBrO}_{3}$, CsA and VALP in RPTEC/TERT1 cells. BBOX1, CLDN2, ATP1B1, MUC1 and AMIGO2 were for the most part decreased by the compound panel. Since these five markers are highly expressed in kidney cells, their decrease may reflect a compound-induced dedifferentiation of the proximal tubule phenotype. ISZ, however, induced BBOX1, CLDN2, ATP1B1 and MUC1, whereas VALP induced ATP1B1, indicating a somewhat more complicated interpretation for certain compounds.

In summary, RPTEC/TERT1 and HepaRG have similar sensitivities to the chosen compounds and exhibit both common and cell type-specific responses. $\mathrm{KBrO}_{3}$ as expected had a clear activation of the Nrf2 and p53 pathways. Whereas, CsA and APAP were strong inducers of the ATF4 pathway. AFP and EREG, were often upregulated upon exposure in HepaRG cells, while A2M, ARG1, BAAT, HNF4A, ADH1A and HPR were most often down regulated. In RPTEC/TERT1 cells, SPP1, APP, SLC7A5 and ME1 were commonly induced, while BBOX-1, CLDN2, ATP1B1, MUC1 and AMIGO2 were often decreased. SLC7A5 and ME1 appear to be renal-specific pathway reporters of ATF4 and $\mathrm{Nrf} 2$, respectively.

In conclusion, this study shows that TempO-Seq is a robust and sensitive method to quantify chemical-induced transcriptomic alterations and also highlights the usefulness of quantitative transcriptomics to identify mechanistic and tissues-specific effects of chemicals.

Acknowledgements The study was funded by the 7th Framework project DETECTIVE (Grant no. 266838 to PJ, BvdW, MV), the Horizon
2020 project EU-ToxRisk (http://www.eu-toxrisk.eu/ Grant no. 681002, to BvdW, PJ), the European Research Council (ERC Starting Grant 335476 to MV), the Center for Alternatives to Animal Testing at Johns Hopkins University Baltimore-USA (to MV), the Tiroler Wissenschaftsfonds (Grant no. UNI-0404/1768, to AW) and the Long Range Initiative Innovative Science Award of the European Chemical Industry Council (CEFIC, 2015 to AL). We would like to thank Pranika Singh for helping with data processing.

\section{Compliance with ethical standards}

Conflict of interest The co-authors P. Shepard, B. Seligmann, H. C. VanSteenhouse and J. M. Yeakley are owners/employees of BioSpyder Technologies Inc., the manufacturer of the TempO-Seq assay. All biological experiments and data interpretation was done independently of BioSpyder. None of the other co-authors are associated with this company financially or otherwise.

Open Access This article is distributed under the terms of the Creative Commons Attribution 4.0 International License (http://creativeco mmons.org/licenses/by/4.0/), which permits unrestricted use, distribution, and reproduction in any medium, provided you give appropriate credit to the original author(s) and the source, provide a link to the Creative Commons license, and indicate if changes were made.

\section{References}

Amundsen R, Asberg A, Ohm IK, Christensen H (2012) Cyclosporine A- and tacrolimus-mediated inhibition of CYP3A4 and CYP3A5 In Vitro. Drug Metab Dispos 40:655-661. https://doi.org/10.1124/ dmd.111.043018

Aschauer L, Gruber LN, Pfaller W et al (2013) Delineation of the key aspects in the regulation of epithelial monolayer formation. Mol Cell Biol 33:2535-2550. https://doi.org/10.1128/ MCB.01435-12

Aschauer L, Limonciel A, Wilmes A et al (2015) Application of RPTEC/TERT1 cells for investigation of repeat dose nephrotoxicity: a transcriptomic study. Toxicol Vitr 30:106-116. https ://doi.org/10.1016/j.tiv.2014.10.005

Bessems JGM, Vermeulen NPE (2001) Paracetamol (acetaminophen)-induced toxicity: molecular and biochemical mechanisms, analogues and protective approaches. Crit Rev Toxicol 31:55-138. https://doi.org/10.1080/20014091111677

Biopredic International (2017) Differentiated HepaRG @ cells cryopreserved: description and user guide for thawing, culture and use. Grégoire, Fr HepaRG, hepatic stem cell line. https ://www.heparg.com/vars/fichiers/Products/HepaRG_differenti ated_User_Guide.pdf

Boesch-Saadatmandi C, Loboda A, Jozkowicz A et al (2008) Effect of ochratoxin A on redox-regulated transcription factors, antioxidant enzymes and glutathione-S-transferase in cultured kidney tubulus cells. Food Chem Toxicol 46:2665-2671. https:// doi.org/10.1016/j.fct.2008.04.023

Cavin C, Delatour T, Marin-Kuan M et al (2006) Reduction in antioxidant defenses may contribute to ochratoxin A toxicity and carcinogenicity. Toxicol Sci 96:30-39. https://doi.org/10.1093/ toxsci/kfl169

Cerveny L, Svecova L, Anzenbacherova E et al (2007) Valproic acid induces CYP3A4 and MDR1 gene expression by activation of constitutive androstane receptor and pregnane $\mathrm{X}$ receptor pathways. Drug Metab Dispos 35:1032-1041. https://doi. org/10.1124/dmd.106.014456 
Chang TKH, Abbott FS (2006) Oxidative stress as a mechanism of valproic acid-associated hepatotoxicity. Drug Metab Rev 38:627-639. https://doi.org/10.1080/03602530600959433

Dadarkar SS, Fonseca LC, Mishra PB et al (2010) Phenotypic and genotypic assessment of concomitant drug-induced toxic effects in liver, kidney and blood. J Appl Toxicol. https://doi. org/10.1002/jat.1562

Dawkins E, Small DH (2014) Insights into the physiological function of the $\beta$-amyloid precursor protein: beyond Alzheimer's disease. J Neurochem 129:756-769. https://doi.org/10.1111/jnc.12675

DeAngelo AB, George MH, Kilburn SR et al (1998) Carcinogenicity of potassium bromate administered in the drinking water to male B6C3F1 mice and F344/N rats. Toxicol Pathol 26:587594. https://doi.org/10.1177/019262339802600501

Doktorova TY, Yildirimman R, Vinken M et al (2013) Transcriptomic responses generated by hepatocarcinogens in a battery of liver-based in vitro models. Carcinogenesis 34:1393-1402. https://doi.org/10.1093/carcin/bgt054

Feierman DE, Melnikov Z, Zhang J (2002) The Paradoxical Effect of Acetaminophen on CYP3A4 Activity and Content in Transfected HepG2 Cells. Arch Biochem Biophys 398:109-117. https ://doi.org/10.1006/abbi.2001.2677

Grimm FA, Iwata Y, Sirenko O et al (2016) A chemical-biological similarity-based grouping of complex substances as a prototype approach for evaluating chemical alternatives. Green Chem 18:4407-4419. https://doi.org/10.1039/C6GC01147K

Guillouzo A, Corlu A, Aninat C et al (2007) The human hepatoma HepaRG cells: a highly differentiated model for studies of liver metabolism and toxicity of xenobiotics. Chem Biol Interact 168:66-73. https://doi.org/10.1016/j.cbi.2006.12.003

Han J, Back SH, Hur J et al (2013) ER-stress-induced transcriptional regulation increases protein synthesis leading to cell death. Nat Cell Biol 15:481-490. https://doi.org/10.1038/ncb2738

Hassan HM, Guo H, Yousef BA et al (2015) Hepatotoxicity mechanisms of isoniazid: a mini-review. J Appl Toxicol 35:1427-1432. https://doi.org/10.1002/jat.3175

Heidari R, Jafari F, Khodaei F et al (2017) The mechanism of valproic acid-induced fanconi syndrome involves mitochondrial dysfunction and oxidative stress in rat kidney. Nephrology. https://doi. org/10.1111/nep.13012

IARC (1993) Some naturally occurring substances food items and constituents, heterocyclic aromatic amines and mycotoxins. IARC Monogr Eval Carcinog Risks Hum 56:489-521

Iorga A, Dara L, Kaplowitz N (2017) Drug-induced liver injury: cascade of events leading to cell death, apoptosis or necrosis. Int $\mathbf{J}$ Mol Sci 18:1018. https://doi.org/10.3390/ijms18051018

Jennings P, Aydin S, Bennett J et al (2009) Inter-laboratory comparison of human renal proximal tubule (HK-2) transcriptome alterations due to cyclosporine A exposure and medium exhaustion. Toxicol Vitr 23:486-499. https://doi.org/10.1016/j.tiv.2008.12.023

Jennings P, Limonciel A, Felice L, Leonard MO (2013) An overview of transcriptional regulation in response to toxicological insult. Arch Toxicol 87:49-72. https://doi.org/10.1007/s00204-012-0919-y

Ke N, Wang X, Xu X, Abassi YA (2011) The xCELLigence system for real-time and label-free monitoring of cell viability. Methods Mol Biol 740:33-43. https://doi.org/10.1007/978-1-61779-108-6_6

Kho D, MacDonald C, Johnson R et al (2015) Application of xCELLigence RTCA biosensor technology for revealing the profile and window of drug responsiveness in real time. Biosensors 5:199_ 222. https://doi.org/10.3390/bios5020199

Komulainen T, Lodge T, Hinttala R et al (2015) Sodium valproate induces mitochondrial respiration dysfunction in HepG2 in vitro cell model. Toxicology 331:47-56. https://doi.org/10.1016/j. tox.2015.03.001

Kustermann S, Boess F, Buness A et al (2013) A label-free, impedance-based real time assay to identify drug-induced toxicities and differentiate cytostatic from cytotoxic effects. Toxicol In Vitro 27:1589-1595. https://doi.org/10.1016/j.tiv.2012.08.019

Li H, Durbin R (2009) Fast and accurate short read alignment with Burrows-Wheeler transform. Bioinformatics 25:1754-1760. https ://doi.org/10.1093/bioinformatics/btp324

Limonciel A, Jennings P (2013) A review of the evidence that ochratoxin A is an Nrf2 inhibitor: implications for nephrotoxicity and renal carcinogenicity. Toxins (Basel) 6:371-379. https://doi. org/10.3390/toxins6010371

Limonciel A, Aschauer L, Wilmes A et al (2011) Lactate is an ideal non-invasive marker for evaluating temporal alterations in cell stress and toxicity in repeat dose testing regimes. Toxicol Vitr 25:1855-1862. https://doi.org/10.1016/j.tiv.2011.05.018

Limonciel A, Wilmes A, Aschauer L et al (2012) Oxidative stress induced by potassium bromate exposure results in altered tight junction protein expression in renal proximal tubule cells. Arch Toxicol 86:1741-1751. https://doi.org/10.1007/s0020 4-012-0897-0

Limonciel A, Moenks K, Stanzel S et al (2015) Transcriptomics hit the target: monitoring of ligand-activated and stress response pathways for chemical testing. Toxicol In Vitro 30:7-18. https://doi. org/10.1016/j.tiv.2014.12.011

Love MI, Huber W, Anders S (2014) Moderated estimation of fold change and dispersion for RNA-seq data with DESeq2. Genome Biol 15:550. https://doi.org/10.1186/s13059-014-0550-8

Marvel SW, To K, Grimm FA et al (2018) ToxPi graphical user interface 2.0: dynamic exploration, visualization, and sharing of integrated data models. BMC Bioinf19:80. https://doi.org/10.1186/ s12859-018-2089-2

Mav D, Shah RR, Howard BE et al (2018) A hybrid gene selection approach to create the $\mathrm{S} 1500$ + targeted gene sets for use in highthroughput transcriptomics. PLoS One 13:e0191105. https://doi. org/10.1371/journal.pone.0191105

Metushi I, Uetrecht J, Phillips E (2016) Mechanism of isoniazidinduced hepatotoxicity: then and now. Br J Clin Pharmacol 81:1030-1036. https://doi.org/10.1111/bcp.12885

Reif DM, Sypa M, Lock EF et al (2013) ToxPi GUI: an interactive visualization tool for transparent integration of data from diverse sources of evidence. Bioinformatics 29:402-403. https://doi. org/10.1093/bioinformatics/bts686

Riese DJ, Cullum RL (2014) Epiregulin: roles in normal physiology and cancer. Semin Cell Dev Biol 28:49-56. https://doi. org/10.1016/j.semcdb.2014.03.005

Riley T, Sontag E, Chen P, Levine A (2008) Transcriptional control of human p53-regulated genes. Nat Rev Mol Cell Biol 9:402-412. https://doi.org/10.1038/nrm2395

Späth GF, Weiss MC (1997) Hepatocyte nuclear factor 4 expression overcomes repression of the hepatic phenotype in dedifferentiated hepatoma cells. Mol Cell Biol 17:1913-1922

Starokozhko V, Greupink R, van de Broek P et al (2017) Rat precisioncut liver slices predict drug-induced cholestatic injury. Arch Toxicol. https://doi.org/10.1007/s00204-017-1960-7

Terentiev AA, Moldogazieva NT (2013) Alpha-fetoprotein: a renaissance. Tumor Biol 34:2075-2091. https://doi.org/10.1007/s1327 7-013-0904-y

Waldmann T, Rempel E, Balmer NV et al (2014) Design principles of concentration-dependent transcriptome deviations in drugexposed differentiating stem cells. Chem Res Toxicol 27:408-420. https://doi.org/10.1021/tx400402j

Wieser M, Stadler G, Jennings P et al (2008) hTERT alone immortalizes epithelial cells of renal proximal tubules without changing their functional characteristics. AJP Ren Physiol 295:F1365F1375. https://doi.org/10.1152/ajprenal.90405.2008

Willebrords J, Pereira IVA, Maes M et al (2015) Strategies, models and biomarkers in experimental non-alcoholic fatty liver disease 
research. Prog Lipid Res 59:106-125. https://doi.org/10.1016/j. plipres.2015.05.002

Wilmes A, Crean D, Aydin S et al (2011) Identification and dissection of the Nrf2 mediated oxidative stress pathway in human renal proximal tubule toxicity. Toxicol In Vitro 25:613-622. https:// doi.org/10.1016/j.tiv.2010.12.009

Wilmes A, Limonciel A, Aschauer L et al (2013) Application of integrated transcriptomic, proteomic and metabolomic profiling for the delineation of mechanisms of drug induced cell stress. J Proteom 79:180-194. https://doi.org/10.1016/j.jprot.2012.11.022

Wu KC, Cui JY, Klaassen CD (2011) Beneficial role of Nrf2 in regulating NADPH generation and consumption. Toxicol Sci 123:590600. https://doi.org/10.1093/toxsci/kfr183
Yeakley JM, Shepard PJ, Goyena DE et al (2017) A trichostatin A expression signature identified by TempO-Seq targeted whole transcriptome profiling. PLoS One 12:e0178302. https://doi. org/10.1371/journal.pone.0178302

Zhang Y, Qi X, Zheng J et al (2016) High-throughput tag-sequencing analysis of early events induced by ochratoxin A in HepG-2 Cells. J Biochem Mol Toxicol 30:29-36. https://doi.org/10.1002/ jbt.21739 